Please send trade news information and

illustrations to Arveen Bajaj at the BDJ, Nature

Publishing Group, The Macmillan Building,

4-6 Crinan Street, London N1 9XW.

Trade news is provided as a service to readers

using text and images from the manufacturer,

supplier or distributor and does not imply

endorsement by the BDJ. Normal and prudent

research should be exercised before purchase or

use of any product mentioned.

\section{Carefully controlled aspiration}

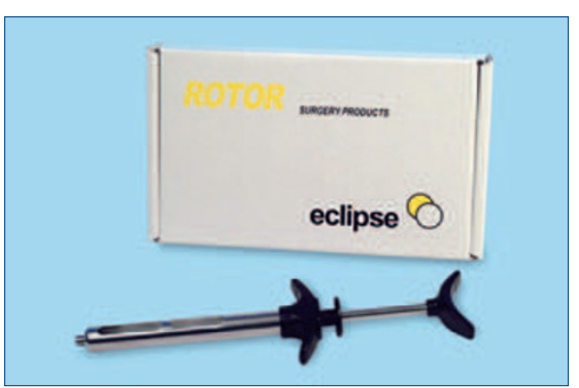

Blackwell Supplies offers a wide range of practical solutions for the dental profession including the effective and easy to use Rotor Syringe range to its customers. Designed to eliminate the use of barbs, the Rotor S/A Syringe uses the elasticity of the cartridge and thumb disk to induce carefully and easily controlled aspiration.

The Rotor Syringe can also be used with the Astra Self-Aspirating Cartridge. This method uses the unique and effective design of the Astra Cartridge to induce aspiration. Designed to be effective and practical, the Rotor Syringe is fully autoclavable, helping dentists to maintain the highest levels of hygiene and infection control within their surgeries.

The moulded components are made from PEEK, offering a lightweight well balanced syringe and also ensuring that components will not rust after repeated exposure to autoclaving or chemicals commonly used within the dental surgery.

New from the range is the $1.8 \mathrm{ml}$ Rotor S/A (self-aspirating) syringe, which has the same construction and benefits of the $2.2 \mathrm{ml}$, but will accept the $1.8 \mathrm{ml}$ anaesthetic cartridges entering the UK dental market. Also the imperial thread on the Rotor $1.8 \mathrm{ml}$ syringe will accept the imperial thread hub needles currently sold through out the UK and Ireland.

The Rotor Syringe is constructed from quality stainless steel, making it exceptionally comfortable to use and ensuring that even after autoclaving, your equipment looks professional and new.

Reader response numbers 53

\section{Colour coded pockets}

Admor has introduced a new distinctive blue colour to their record pocket range. Whatever your requirements, whether an NHS or private practice, Admor has a wide range of solutions that are designed specifically to help you run your practice more effectively.

The range of record pockets, record cards and covers can be tailored to your practice's individual needs. Available as standard or heavy duty, they additionally can be pre-printed with your own unique

\section{Easy to swallow capsules}

Molar Ltd has announced that ProDen PlaqueOff is now being produced in a new easy to swallow capsule format. ProDen PlaqueOff is particularly useful for those patients who have problems controlling plaque and calculus using traditional methods.

By taking two capsules daily, reductions in calculus, plaque and bad breath is normally seen within five to eight weeks. The patented formulation contains only specially selected seaweed from Norway and Iceland. The new capsules are suitable for vegetarians, easy to swallow and have an improved taste.

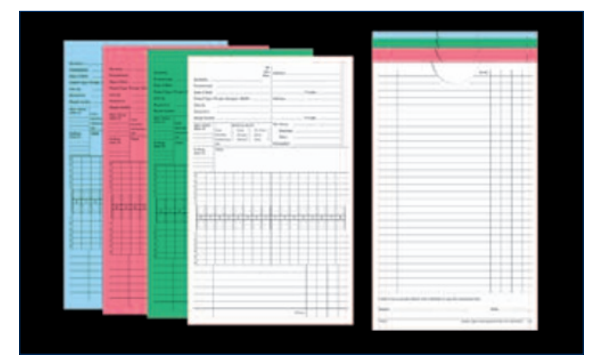

design. Admor is pleased to extend this range, currently available in white, green and pink, by offering more colour choices. Reader response numbers 50

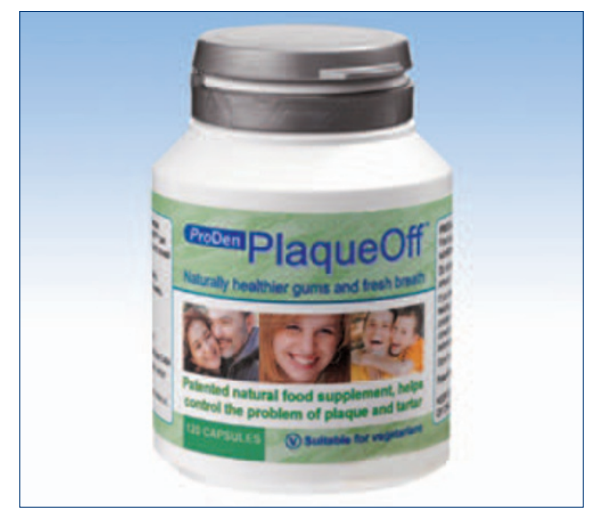

The new capsule format will be available to order from your regular wholesaler (new stock will be phased in as old stock is depleted).

Reader response numbers $\mathbf{5 1}$

\title{
Optical equipment cleaner
}

OptiMax, the Ultra Pure Cleaning Solution, is the multi-purpose cleaning formula that easily removes smears and dirt from all types of optical equipment. OptiMax is an advanced anti-static, non-toxic solution, instantly cleaning perfectly and leaving no visible film or residue behind, enabling your surface area to stay clean longer.

A sterile clear solution packaged in a class 100 clean room, it has a neutral pH and is non-conductive so can be used on live low voltage equipment. It can be used for all optical lens, coated eye-wear, com- puter monitors, LCD and plasma displays, optical disks, cameras, loupes, microscope and video optics, mobile phones and other handhelds, notebooks and more.

OptiMax ensures all compatible surfaces are $100 \%$ particle and dust free. Reader response numbers 52






\section{Looking towards a faster recovery}

Visual Therapy offered by diagnostic imaging specialists MAS Medical Limited, is a range of over 2,000 images by award winning nature photographer, Joey Fischer. In a healthcare setting such as a dental practice, images are used in both clinical and non-clinical areas to promote a healing environment.

The company says research has shown that images of nature can dramatically improve patient stress levels, reduce systolic blood pressure, and minimise boredom - lessening the likelihood of complications and the need for expensive repeat diagnosis or treatment.

Images are usually supplied as backlit transparencies and can be either wall or ceiling mounted, with wall mounted images supplied in custom made light boxes. Ceiling images are configured to fit within the ceiling grid or can be fitted in bespoke light boxes.

MAS Medical offer advice with regards to size, position and image selection. For example, in cancer services, autumn scenes are not usually recommended because this season is often associated with an ending.

Reader response number 55

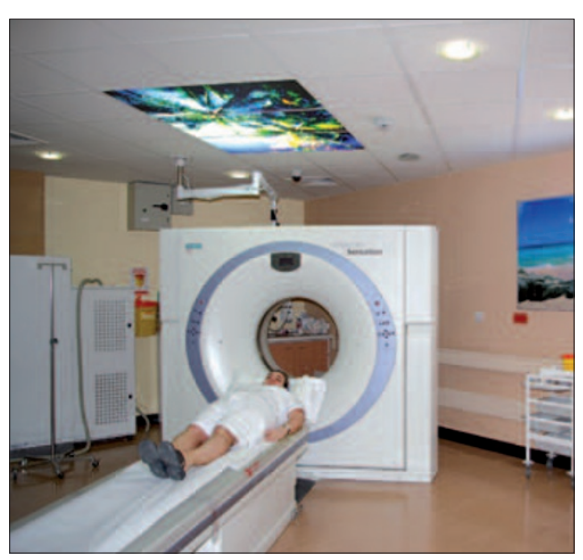

\section{Flexible dental system}

The Clesta II from Takara Belmont is a flexible dental system that comes in four configurations: Over the Patient Treatment Centre; Continental Treatment Centre; Side Arm (Cabinetry Mounted) Treatment Centre and Mobile Cart Treatment Centre. Each configuration can also be ordered in 'Air' and 'Electric' mode for increased choice.

The electric E-type offers high specification and technical uplift with fibreoptics, micro motor and digital display all as standard. Every configuration can be ordered in left or right hand format from the UK factory.

To help save time and improve the patients' treatment experience, the Clesta II comes with a cuspidor that rotates through $180^{\circ}$ to allow the rinsing bowl to be positioned over the patient or out of the way for clear access for the nurse. The cuspidor also comes with an easy to remove detachable glass bowl to improve hygiene and speed of cleaning.

\section{Fully integrated digital unit}

The I-Max digital panoramic unit, available exclusively in the UK from Imaging Technologies is a high tech piece of equipment that can be used for panoramic radiographs of adults as well as children, with the mouth open or closed and is useful to X-ray the sinuses.

Fully integrated with Quick Vision software, the I-Max is simple to operate, offering effective diagnoses based on digital technology and Owandy image processing. With the image being digitised at source, loss of clarity is eliminated as is the problem of information becoming altered or damaged. Sharing resources with I-Max is simple

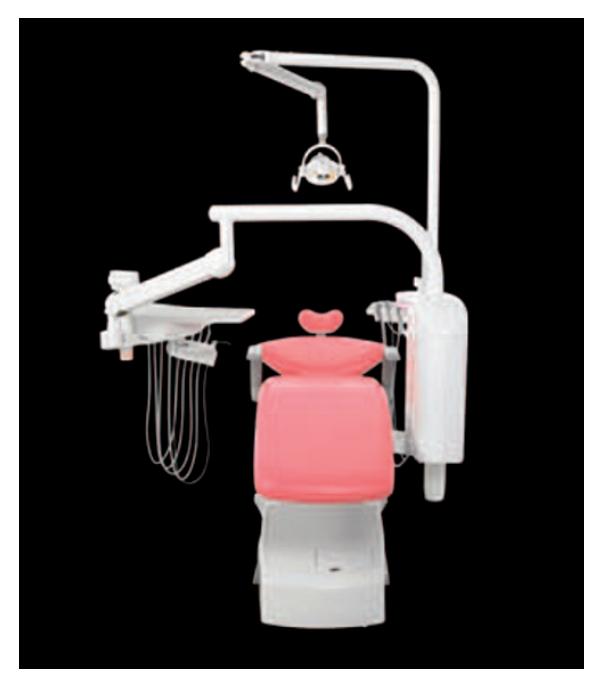

The lightweight, over-the-patient swing arm is engineered to provide effortless movement and precision positioning. Each Clesta II comes with a state of the art Belmont 720 dental light that incorporates with a composite safe mode as standard to increase working time. Reader response number 54

regardless of your current configuration. The USB 2.0 interface of the I-Max can be easily connected to any PC with a USB port.

The I-Max Easy is supplied with a Compact Flash card that can be used to store images without a PC connection. The removable card can then be taken to any other PC in the practice or off the premises.

It offers precision of diagnosis, reverse video and pseudo-colours, you may zoom in or out, and the Quick Vision software can manipulate the image in ways that help you better understand the patient's pathology. Going digital will cut out developing costs and associated pollutants, and free up the space previously occupied by the darkroom.

Reader response number 56 


\section{A professional impression}

Designer Dental is a specialist dental marketing company that offer a full marketing service to dental practices, from the design of a practice logo through to patient welcome packs, newsletters, posters and marketing.

It can produce eye-catching, professional and quality referral packs that will reflect the services that you offer. Referral forms, newsletters, fee guides, referral information and reply envelopes are all housed in folders with expert finishes such as matt lamination, spot UV

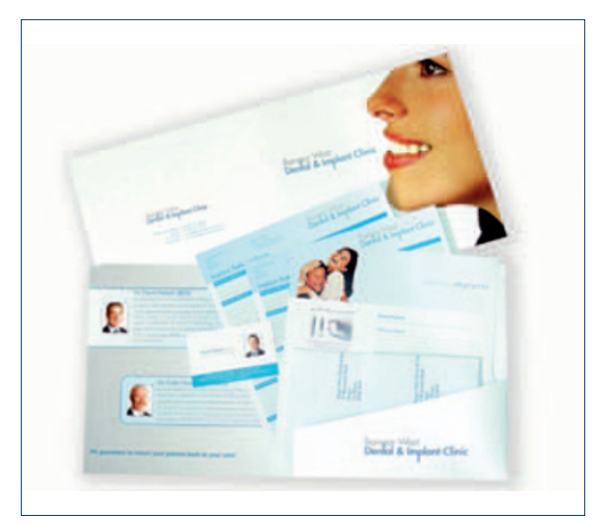

varnish or embossing - all giving an organised, professional impression.

Reader response number 57

\section{Help with changing storage requirements}

The Variant D drawer system has recently been launched with the new laboratory and dental furniture fittings and accessories catalogue from Häfele. Variant D drawers are designed for use in conjunction with dental and laboratory dedicated samples, components and instruments around a range of plastic coated moulded inserts.

Drawers are sized in line with $500 \mathrm{~mm}$ width standard domestic (kitchen-style) cabinet carcasses and are mounted on single 3/4 extension runners with $345 \mathrm{~mm}$ travel giving full access to all contents within the $310 \mathrm{~mm}$ depth drawer boxes.

Drawer height options (top-to-bottom depths) of $39 \mathrm{~mm}, 90 \mathrm{~mm}$ and 160 $\mathrm{mm}$ are available, each divided into four or nine compartments or into multidivision instrument/spectacle compartments as well as being offered as plain shells.
The $2.5 \mathrm{~mm}$ thick plastic of Variant D drawer box inserts is peel-off film coated for full protection and hygiene to the point of on-site commissioning. Steel Variant D drawer box inserts are moulded with side skirts to conceal and protect the roller bearing slide mechanisms, each of which is self-closing.

Drawers need only minimal pressure to close - self-closure mechanisms take care of remaining travel and are also available with optionally damped closure to ensure a soft and silent return to the fully closed position. Easily and precisely self-locating on the steel support slides, Variant D drawer boxes require no fixing into position. Differently configured inserts can therefore be easily removed, relocated or interchanged to meet regularly or occasionally changing storage requirements.

Reader response number 58

\section{Fully ambidextrous}

The Pelton and Crane Ellipse Dental unit from Henry Schein provides versatility with comfort. The Ellipse accommodates left or right handed dentists with equal

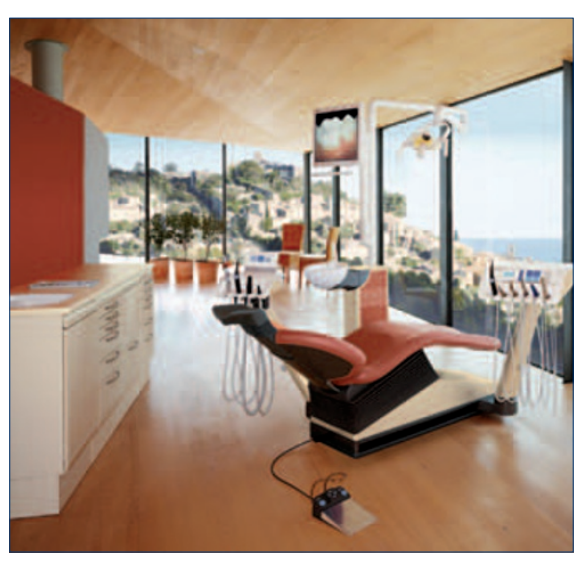

facility as it is fully ambidextrous, the chair also swivels on its axis allowing for perfect specialist treatment, and all chair controls are easily accessible by the operator or the nurse.

The ErgoSoothe fully integrated lumbar and back massaging system, in association with the soft and ultra durable Ultraleather upholstery offers the patient full comfort. The operating light features the feathered-edge pattern to reduce eye fatigue and combines with a triple axis operating light head to allow a wider range of working positions.

The quartz halogen lamp is easily replaced when required via a hinged light back. The Pelton and Crane units offer full flexibility, yet integrating all features required in the modern dental practice.

Reader response number 59 